\title{
A two-scale modelling approach to reaction-diffusion processes in porous materials
}

\author{
S. A. Meier ${ }^{a}$, M. A. Peter ${ }^{\mathrm{a}, 1}$ and M. Böhm ${ }^{\mathrm{a}}$ \\ ${ }^{a}$ Centre for Industrial Mathematics, FB 3, University of Bremen, \\ Postfach 330 440, 28334 Bremen, Germany.
}

\begin{abstract}
A two-scale model for a reaction-diffusion process in a porous material is developed and applied to a simplified problem related to concrete carbonation. The material is exposed to a gas which enters from the outside, diffuses through the air-filled pore space, and dissolves into the pore water where it reacts with a second solute. The problem exhibits two different spatial scales due to the great difference in diffusivities of the gas in air and water and the fine pore geometry. In contrast to common purely macroscopic models, the proposed two-scale model is capable of resolving both scales. It is shown by numerical simulations that different pore geometries lead to different macroscopic output. Results of a comparable purely macroscopic model can be recovered as a special case.
\end{abstract}

PACS: 82.20.Wt, 81.05.Rm, 82.33.Ln, 02.60.Lj, 02.70.Dh

Key words: reaction-diffusion, multiscale, microstructure, unit cell, concrete carbonation

\section{Introduction}

In this paper, we develop a two-scale model for a reaction-diffusion process in an unsaturated porous material. The medium is exposed to a gas which enters the medium from the outside and diffuses through the air-filled pore space. It dissolves into the pore water where it reacts with a second solute. We

$\overline{1}$ Corresponding author. Tel.: +49 421 2189173, fax: +49 421 2189406. E-mail addresses: sebam@math.uni-bremen.de (S. A. Meier), mpeter@math.uni-bremen.de (M. A. Peter), mbohm@math.uni-bremen.de (M. Böhm). 
consider the case where the diffusivity of the gas in air is much greater than in the pore water. Consequently, the problem exhibits two spatial scales: the macroscopic scale, at which the gas diffuses through the air-filled pore space and the microscopic scale, at which the gas diffuses in the pore water and reacts.

A typical process exhibiting this feature is the degradation of reinforced concrete structures induced by carbonation. This process takes place in the pores of the concrete which are partially saturated with water that clings to the pore walls. Atmospheric $\mathrm{CO}_{2}$ enters the concrete through the air-filled pores and gets dissolved in the pore water. There it reacts with dissolved constituents of the cement paste. This causes a lowering of the $\mathrm{pH}$, facilitating the corrosion of the steel reinforcements and, consequently, leads to a severe reduction of the service life of the structure. The dominant carbonation reaction is usually assumed as

$$
\mathrm{CO}_{2}(a q)+\mathrm{Ca}(\mathrm{OH})_{2}(a q) \stackrel{\mathrm{H}_{2} \mathrm{O}}{\longrightarrow} \mathrm{CaCO}_{3}(a q)+\mathrm{H}_{2} \mathrm{O} .
$$

The produced $\mathrm{CaCO}_{3}$ precipitates very quickly to the solid matrix. Detailed surveys on the carbonation problem were carried out, for instance, by Kropp [1], Bier [2], and Chaussadent [3].

Existing models of the carbonation process describe the mass balances of the active species only on the macroscopic scale. We refer to the approaches by Saetta et al. [4], Papadakis et al. [5], and Steffens et al. [6]. The drawback of such pure macromodels is that effects due to the local pore geometry are only captured via few empirical constants such as the tortuosity and porosity factors. The relatively slow diffusion of $\mathrm{CO}_{2}(a q)$ and $\mathrm{Ca}(\mathrm{OH})_{2}(a q)$ in the pore water is usually neglected. A possible delay time caused by reactants not being immediately available is therefore not taken into account. In the present paper, we propose a model which consists of a macroscopic equation coupled with a microscopic system that is solved in every point on a representative unit cell (RUC). Such models have been proposed, mathematically justified, and implemented for flow in naturally fractured reservoirs [7-9]. There are also various two-scale computational approaches of modelling mechanical properties of materials with microstructure $[10,11]$. In this area of application, two-scale simulations have already proven efficient and reliable.

As we are primarily interested in studying the principal effects of such a model, we consider a highly simplified carbonation scenario. In section 2 , we present the two-scale model incorporating the basic reaction-diffusion processes inside the concrete. As a reference, a typical purely macroscopic model based on [5] is also included. In section 3, we present simulation results for the carbonation of a concrete piece based on Ordinary Portland Cement (OPC). We consider two different RUC geometries and point out the differences in the results captured by the two-scale model but not resolved in the correspond- 
ing pure macromodel. In all cases, the problems in the RUC are reduced to one space dimension for computational reasons. However, it should be emphasised that the model is conceptually capable of being extended to a more complete carbonation scenario. Finally, section 4 collects our main results and conclusions.

\section{Mathematical modelling}

In order to fix ideas, we develop the two-scale model for the particular problem of concrete carbonation. In this context, our model is based on the following simplifying assumptions.

(1) The humidity is uniformly distributed in the sample and time-independent.

(2) Dissolution of solid $\mathrm{Ca}(\mathrm{OH})_{2}$ is sufficiently fast and is therefore neglected.

(3) No matrix constituents other than $\mathrm{Ca}(\mathrm{OH})_{2}$ react with $\mathrm{CO}_{2}$.

(4) The pore structure does not change in time.

Experiments show that, in reality, the pore structure is altered by the carbonation process itself. Moreover, the humidity entering the sample from outside or being produced by reaction can have a strong influence on the carbonation [6]. Nevertheless, we neglect these processes in this first approach and concentrate on the basic features of the two-scale model.

\subsection{The two-scale model}

We start with the representation of the local pore geometry. Let $x=\left(x_{1}, x_{2}, x_{3}\right)$ be a point of the concrete sample $\Omega$. We assume the local pore geometry at $x$ to be described by a representative unit cell (RUC) contained in a cuboid $Y$, as depicted in figure 1 . In $Y$, a local coordinate system $y=\left(y_{1}, y_{2}, y_{3}\right)$ is introduced. We refer to $y$ as the microscopic variable, in contrast to the macroscopic variable $x$. Three different phases are considered: the air-filled pore space $Z^{\mathrm{a}}$, the water-filled pore space $Z^{\mathrm{w}}$ and the solid matrix $Z^{\mathrm{s}}$. The air-water and water-solid interfaces are denoted by $\Gamma^{\mathrm{a}}$ and $\Gamma^{\mathrm{s}}$, respectively.

The transport of the dissolved species in the pore water is comparably slow and therefore restricted to the local RUC. We denote the mass concentration of $\mathrm{CO}_{2}(a q)$ by $u_{2}(x, y, t)$ and that of $\mathrm{Ca}(\mathrm{OH})_{2}(a q)$ by $u_{3}(x, y, t)$. The mass balances read as

$$
\begin{array}{ll}
\partial_{t} u_{2}-D_{2} \Delta_{y} u_{2}=-m_{2} R\left(u_{2}\right)^{p}\left(u_{3}\right)^{q}, & x \in \Omega, y \in Z^{\mathrm{w}}, \\
\partial_{t} u_{3}-D_{3} \Delta_{y} u_{3}=-m_{3} R\left(u_{2}\right)^{p}\left(u_{3}\right)^{q}, & x \in \Omega, y \in Z^{\mathrm{w}},
\end{array}
$$




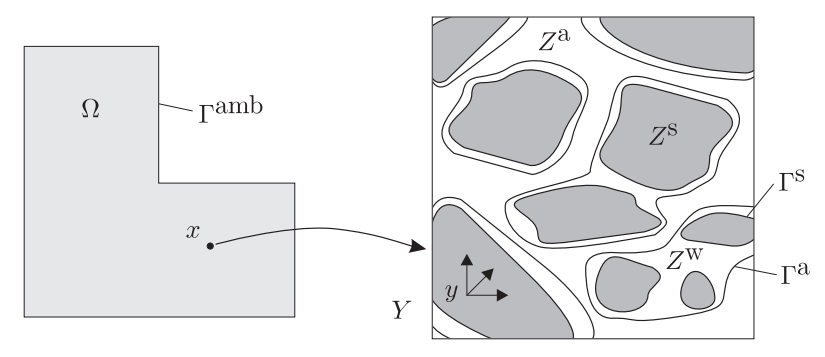

Fig. 1. A cross-section of a corner of a concrete structure, together with a typical RUC around a point $x$ of the medium.

where the diffusivities $D_{2}$ and $D_{3}$ are of the same magnitude. Here $\Delta_{y}$ denotes the Laplace operator where the derivatives are taken with respect to $y$. The macroscopic variable $x$ serves only as a parameter in (1a) and (1b). To describe the reaction kinetics, we adopt a generalised form of the law used in [6]. The quantities $m_{2}=44 \mathrm{~g} / \mathrm{mol}$ and $m_{3}=74 \mathrm{~g} / \mathrm{mol}$ are the molar weights of $\mathrm{CO}_{2}$ and $\mathrm{Ca}(\mathrm{OH})_{2}$, respectively, whereas $R, p$, and $q$ are empirical constants, Typical values are $p=q=1$ and $R=160 \mathrm{~mol} \cdot \mathrm{cm}^{3} /\left(\mathrm{g}^{2} \mathrm{~d}\right)[6]$.

The exchange of $\mathrm{CO}_{2}$ with the air phase in the pores is described by Henry's law. This leads to the boundary condition at the air-water interface $\Gamma^{\mathrm{a}}$,

$$
-D_{2} \nabla_{y} u_{2} \cdot \nu=-K\left(H u_{1}-u_{2}\right), \quad y \in \Gamma^{\mathrm{a}},
$$

where $u_{1}(x, t)$ is the mass concentration of $\mathrm{CO}_{2}(g)$ in air, $H=0.81$ is the Henry constant for $\mathrm{CO}_{2}$ in water [12], and $\nu$ is the outward unit normal at $\Gamma^{\mathrm{a}}$. Therefore, the microscopic system is closed by the no-flux conditions

$$
-D_{2} \nabla_{y} u_{2} \cdot \nu=0, \quad y \in \Gamma^{\mathrm{s}} ; \quad-D_{3} \nabla_{y} u_{3} \cdot \nu=0, \quad y \in \Gamma^{\mathrm{a}} \cup \Gamma^{\mathrm{s}},
$$

and initial conditions for $u_{2}$ and $u_{3}$, i.e. $u_{2}(0)=0$ and $u_{3}(0)=u_{3}^{0}$.

Diffusion of $\mathrm{CO}_{2}(g)$ in air is fast compared to diffusion in water. The mass balance for the mass concentration $u_{1}$ of $\mathrm{CO}_{2}(g)$ is therefore approximated by an averaged equation of the form

$$
\theta^{\mathrm{a}} \partial_{t} u_{1}-\theta^{\mathrm{a}} \tau^{\mathrm{a}} D_{1} \Delta u_{1}=-\frac{1}{|Y|} \int_{\Gamma^{\mathrm{a}}} K\left(H u_{1}-u_{2}\right) \mathrm{d} y, \quad x \in \Omega .
$$

Here $|Y|$ denotes the volume of $Y$. The effective diffusion coefficient for $\mathrm{CO}_{2}(g)$ is a product of the volume fraction of the gaseous phase $\theta^{\mathrm{a}}=\left|Z^{\mathrm{a}}\right| /|Y|$, the diffusivity $D_{1}$ of $\mathrm{CO}_{2}(g)$ in air, and a tortuosity factor $\tau^{\mathrm{a}}$. Consequently, the mass concentration $u_{1}$ refers to the volume of the air phase. The integral term on the right-hand side of (1e) gives the total flux inside the RUC (1c) passing through the air-water interface at time $t$. Note that, by (1e) and (1c), the macroscopic and the microscopic systems are coupled. Finally, at the exposed outer boundary $\Gamma^{\mathrm{amb}}$ of the sample $\Omega$, the exchange with the environment is 
described by

$$
-\theta^{\mathrm{a}} \tau^{\mathrm{a}} D_{1} \nabla_{y} u_{1} \cdot \nu=B\left(u_{1}-u_{1}^{\mathrm{amb}}\right), \quad x \in \Gamma^{\mathrm{amb}},
$$

where $u_{1}^{\mathrm{amb}}$ is the ambient $\mathrm{CO}_{2}$ concentration.

\subsection{The macroscopic reference model}

As a reference for the two-scale model, we use a simplified version of the model introduced in [13]. This classic purely macroscopic model is based on the models in [5] and [6] and reads as

$$
\begin{aligned}
\theta^{\mathrm{a}} \partial_{t} u_{1}-\theta^{\mathrm{a}} \tau^{\mathrm{a}} D_{1} \Delta u_{1} & =-\frac{\left|\Gamma^{\mathrm{a}}\right|}{|Y|} K\left(H u_{1}-u_{2}\right), \\
\theta^{\mathrm{w}} \partial_{t} u_{2}-\theta^{\mathrm{w}} \tau^{\mathrm{w}} D_{2} \Delta u_{2} & =\frac{\left|\Gamma^{\mathrm{a}}\right|}{|Y|} K\left(H u_{1}-u_{2}\right)-\theta^{\mathrm{w}} m_{2} R\left(u_{2}\right)^{p}\left(u_{3}\right)^{q}, \\
\theta^{\mathrm{w}} \partial_{t} u_{3}-\theta^{\mathrm{w}} \tau^{\mathrm{w}} D_{3} \Delta u_{3} & =-\theta^{\mathrm{w}} m_{3} R\left(u_{2}\right)^{p}\left(u_{3}\right)^{q},
\end{aligned}
$$

together with the initial conditions and the boundary condition (1f). All concentrations in (2) are functions of $x$ and $t$ only. The local geometry only enters the system (2) via the constants $\left|\Gamma^{\mathrm{a}}\right|,|Y|$, the volume fractions $\theta^{\mathrm{a}}$ and $\theta^{\mathrm{w}}$, and the tortuosity factors of the gaseous and liquid phases, $\tau^{\mathrm{a}}$ and $\tau^{\mathrm{w}}$.

It should be noticed that both models, (1) and (2), can be derived rigorously from virtually the same micromodel by the mathematical homogenisation method (see [9] and references therein). The type of the homogenised model depends on the relation of the time scales of the involved processes; see $[14,7]$ for the derivation of a two-scale model and [15] for that of a pure macromodel. This yields a mathematical justification of the validity and comparability of both models.

\section{Simulation results}

Both the two-scale model (1) and the macromodel (2) are numerically approximated using the finite element method with linear test and trial functions. The macroscopic domain $\Omega$ (cf. fig. 1) is always discretised by a constant uniform mesh, the macroscopic mesh. For the two-scale model, in every grid node of the macroscopic mesh we need to solve the weakly-coupled system of parabolic equations (1a)-(1d) in the RUC, more precisely in $Z^{\mathrm{w}}$. In order to avoid expensive three-dimensional computations, simplified RUC geometries are considered allowing the reduction of the cell problem to one space dimension. Therefore, the microscopic mesh is one-dimensional. Two highly 
different RUC geometries are chosen in order to show their influence on the results. They are described in detail below. The resulting system of ordinary differential equations in time is solved using a standard implicit solver.

\subsection{Carbonation of an OPC concrete sample}

The size of the RUC should be chosen in the range of a typical capillary pore diameter. For OPC-based concrete this quantity can vary between $10^{-7} \mathrm{~cm}$ and $10^{-4} \mathrm{~cm}[5]$. For the present simulations, we use $\ell=10^{-4} \mathrm{~cm}$ as a characteristic microscopic length and choose the RUC size such that after the change of variables $\bar{y}=y / \ell$, the transformed cuboid $Y$ has unit volume $|Y|=1$. In the special case of a cube, we therefore have $Y=(0,1)^{3}$. The geometry of the RUC refers to a rotationally symmetric microstructure (fig. 2, left). A spherical matrix ball is surrounded by a liquid film. The volume fractions are $\theta^{\mathrm{a}}=0.22$ and $\theta^{\mathrm{w}}=0.1$. For the tortuosity factors, we choose the empirical values $\tau^{\mathrm{a}}=3 \cdot 10^{-3}$ and $\tau^{\mathrm{w}}=10^{-3}$. According to the assumption of a uniform pore structure and a uniform pore water distribution, the same RUC is chosen throughout the whole concrete sample. It has to be noted that this choice of the RUC is a rather strong simplification. As is illustrated in [2], e.g., the real pore configuration of concrete is much more complicated than the RUC structure used here. However, it is sufficient to observe essential features of the model.

The values of the remaining model parameters refer to an L-shaped crosssection of an OPC concrete sample with a side length of $5 \mathrm{~cm}$ under natural exposure conditions. Our simulations cover a period of 16 years and begin after the curing time. At this time, we assume no $\mathrm{CO}_{2}$ inside the concrete sample and that $0.07 \mathrm{~g} / \mathrm{cm}^{3}$ of $\mathrm{Ca}(\mathrm{OH})_{2}$ is available to the carbonation reaction. The ambient $\mathrm{CO}_{2}$ concentration is $0.54 \cdot 10^{-6} \mathrm{~g} / \mathrm{cm}^{3}$. The diffusion coefficient of $\mathrm{CO}_{2}(g)$ is chosen as $D_{1}=9.6 \cdot 10^{3} \mathrm{~cm}^{2} / \mathrm{d}[5,6]$. The diffusivities of the ions in water are $D_{2}=D_{3}=1 \mathrm{~cm}^{2} / \mathrm{d}[16]$.
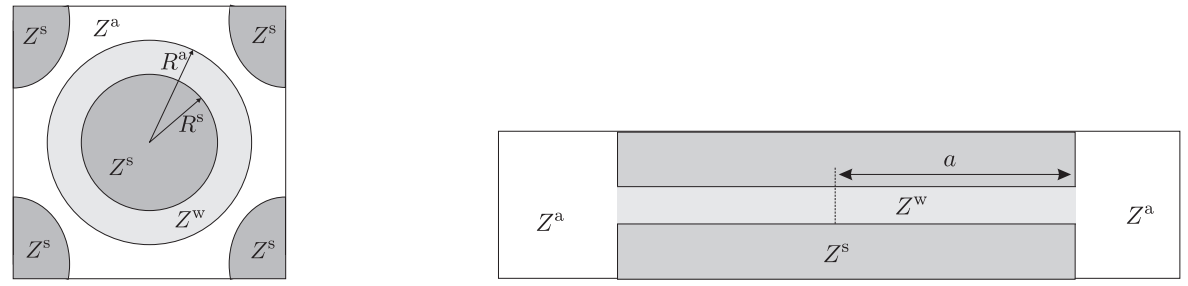

Fig. 2. Schematic cross-sections of the RUCs used in the simulations. Left: spherical geometry; $R^{\mathrm{s}}=0.44 \ell ; R^{\mathrm{a}}=0.48 \ell$. Right: pore channel of length $2 a=6 \ell$. The cross-sectional area of the channel is $0.017 \ell^{2}$, where $\ell=10^{-4} \mathrm{~cm}$.

In figure 3 , results of the two-scale model with the spherical pore geometry are presented. The concentration profile of $u_{3}$ is a normalised volume average with 
respect to the microscopic variable $y$; more precisely, the quantity $\int_{Z^{\mathrm{w}}} u_{3} \mathrm{~d} y / u_{3}^{0}$ is plotted. It can be observed that the bulk of the carbonation reaction is concentrated on a narrow zone which advances into the concrete with time, and the $\mathrm{Ca}(\mathrm{OH})_{2}$-concentration exhibits steep gradients near the reaction zone due to the low diffusivity of $\mathrm{Ca}(\mathrm{OH})_{2}$. This coincides with other simulation results [5,13]. The plot of the concentration profile of $\mathrm{CO}_{2}(a q)$ is not shown since it is visually identical to that of $\mathrm{CO}_{2}(g)$ due to the fast absorption and fast diffusion of $u_{2}$ across the thin water film $Z^{\mathrm{w}}$. This can also be read off the cell solutions (not shown): the microscopic (spatial) profiles of $u_{2}$ and $u_{3}$ within every cell are nearly constant at each instant in time.

In figure 4, one-dimensional cuts of the profiles are compared with results from the macromodel using the same parameters. The cut is specified in figure 3 (left). The results are very similar, undermining the validity of the twoscale approach. An important quantity for durability issues is the carbonation depth, i.e. the depth how far the carbonation zone has penetrated into the concrete sample after a given amount of time. ${ }^{2}$ In figure 5 , the carbonation depth predicted by the two-scale model and by the macromodel are plotted in comparison to experimental data by Wierig [17] (long-term exposure out of doors under roof). The simulated curves exhibit the same tendency as the measured values except for an initial delay time. This effect might be caused by the unrealistic model assumption that at $t=0$ the carbonation has not yet started.
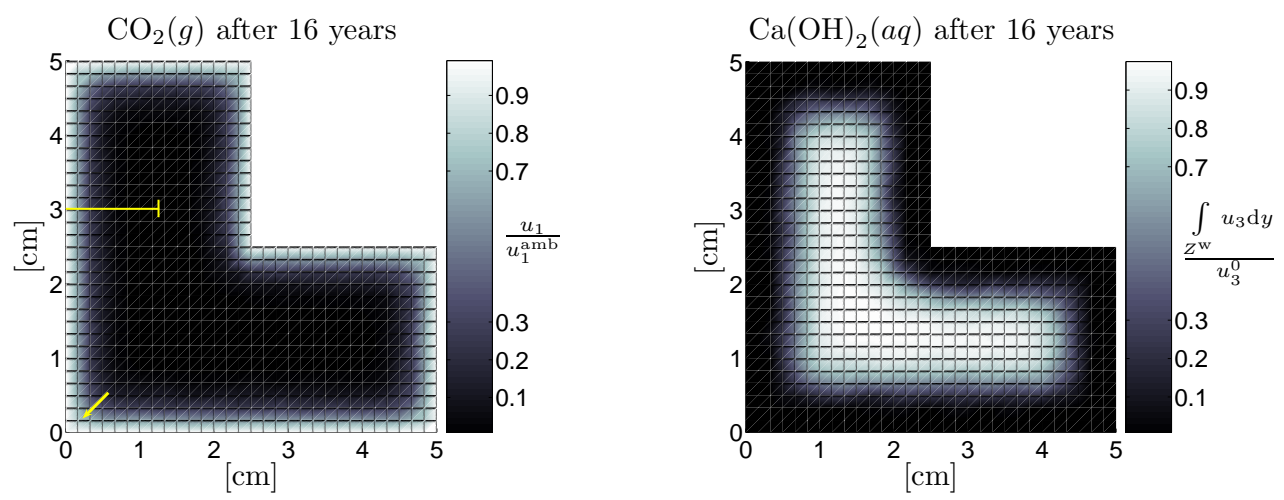

Fig. 3. Dimensionless concentration profiles after 16 years obtained with the two-scale model using the spherical pore geometry. Left: $\mathrm{CO}_{2}(g)$; right: $\mathrm{Ca}(\mathrm{OH})_{2}(a q)$. In the left plot, the cut and the cell are depicted for which the solutions are presented in figures 4, 6 and 7 below.

$\overline{2}$ Equivalently to Steffens [6] et al., we define the carbonation front as the level set $\int_{Z^{\mathrm{w}}} u_{3} \mathrm{~d} y / u_{3}^{0}=0.1$. 

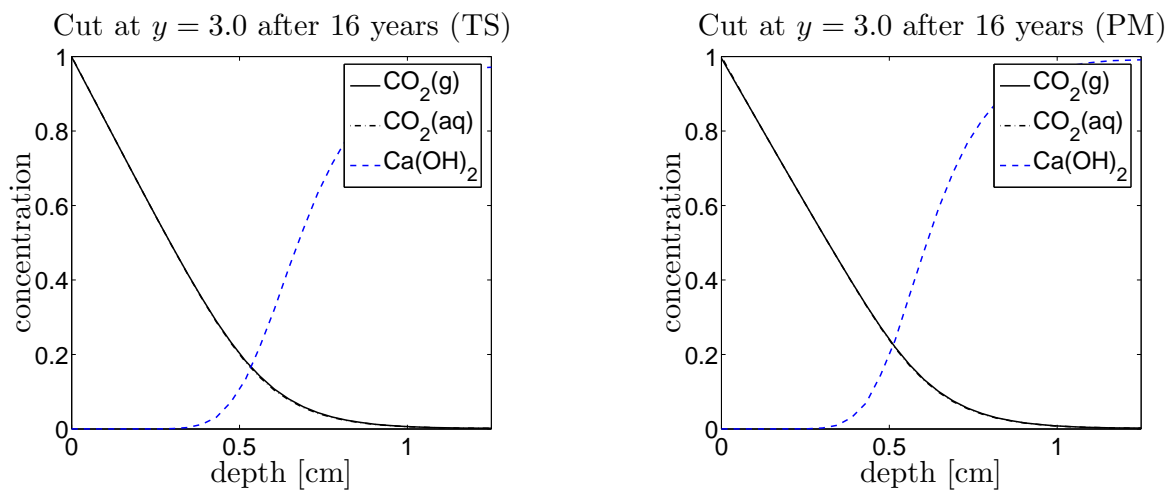

Fig. 4. Concentration profiles obtained using the spherical pore geometry with the two-scale model (left) and the pure macromodel (right). (c) Carbonation depth versus time compared to experimental data by Wierig [17].

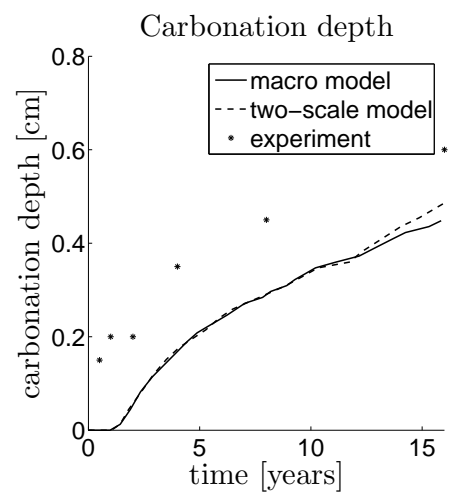

Fig. 5. Carbonation depth versus time obtained using the spherical pore geometry compared to experimental data by Wierig [17].

\subsection{Pore-scale effects}

Now we focus on the main feature of the two-scale model to resolve effects on the pore scale. It is expected that processes on the microscale affect the macroscopic behaviour if their characteristic time is sufficiently large. Therefore, we test our model with a slightly different parameter setting: We prescribe the RUC geometry as a narrow pore channel of a characteristic length $2 a$ that is saturated with pore water (fig. 2, right). The volume fractions are again $\theta^{\mathrm{a}}=0.22$ and $\theta^{\mathrm{w}}=0.1$. Due to the narrowness of the channel, diffusion perpendicular to the channel length is assumed neglegible, and the cell system is again reduced to a one-dimensional problem. Taking into account the symmetry of the channel, it suffices to compute the solutions on the interval $(0, a)$. Moreover we assume a slower transport of the species in the pore water, i.e. $D_{2}=D_{3}=10^{-6} \mathrm{~cm}^{2} /$ d. The tortuosity factors $\tau^{\mathrm{a}}=3 \cdot 10^{-3}$ and $\tau^{\mathrm{w}}=10^{-3}$ are the same as before. If more complex pore geometries are used, it can be advantageous to use averaging theories for adapting the tortuosities to the cell geometry. This should be possible by homogenisation or percolation theory. 
We remark that the spherical geometry has also been tested with slower diffusivities of $u_{2}$ and $u_{3}$. Down to values in the range of $10^{-6} \mathrm{~cm}^{2} / \mathrm{d}$, the simulation profiles (figs. 3 and 4) remain almost unchanged.

In figures 6 and 7, results obtained with the pore-channel geometry and the slower transport are shown. The concentration profiles for the purely macroscopic model are almost the same as for the spherical geometry (fig. 6, right). For the two-scale model, highly different results are obtained. The $\mathrm{CO}_{2}$ penetrates much deeper into the material (fig. 6, left) and the carbonation progress is more advanced. The cell profiles of $\mathrm{CO}_{2}(a q)$ are not horizontal (fig. 7, left). This can be seen as an effect due to the relatively long pore channel and the slow diffusion of $\mathrm{CO}_{2}(a q)$. The inhomogeneous $\mathrm{CO}_{2}(a q)$ profile and the smaller interfacial area $\left|\Gamma^{\mathrm{a}}\right|$ cause a lower absorption of $\mathrm{CO}_{2}(g)$ in the pore water and therefore a higher penetration of $\mathrm{CO}_{2}(g)$. We emphasise that this result does not seem to be realistic for the concrete-carbonation problem. However, it can be clearly seen that if the transport processes on the microscale are sufficiently slow, then the specific pore structure of the material can have a large impact on the macroscopic process. This effect can not be captured by the corresponding purely macroscopic model.
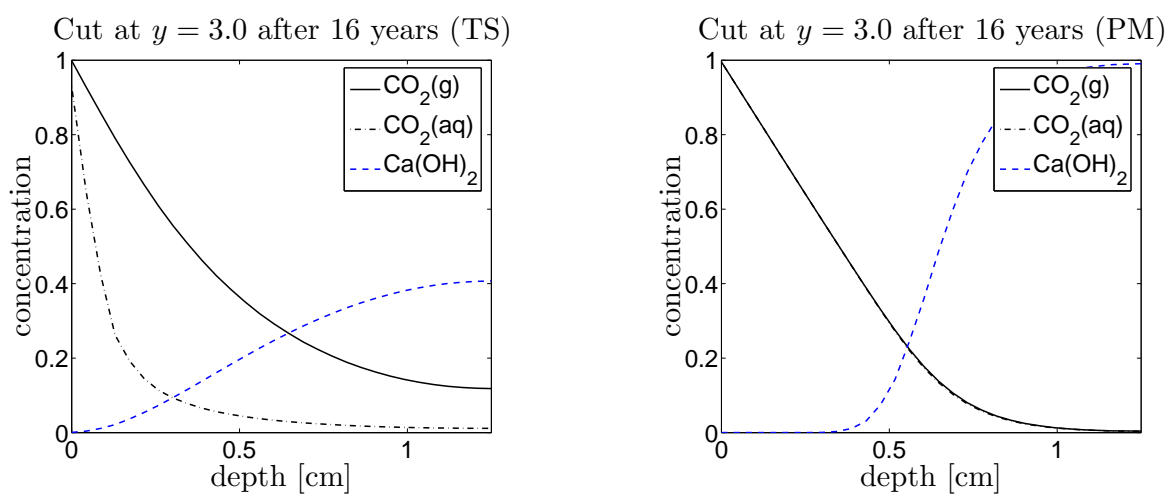

Fig. 6. Concentration profiles obtained using the pore-channel geometry with (a) the two-scale model and (b) the pure macromodel.
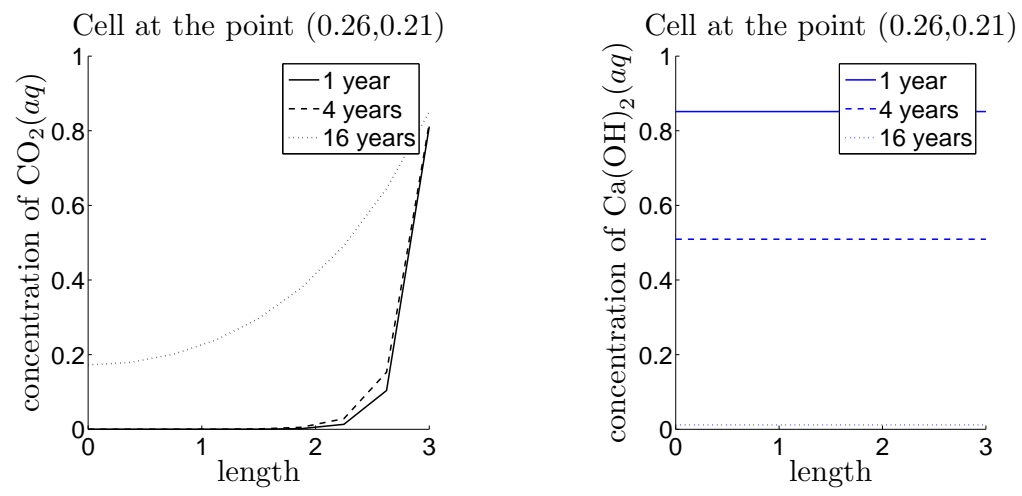

Fig. 7. Cell solutions within one particular RUC obtained with the two-scale model using the pore-channel geometry. (a) $\mathrm{CO}_{2}(a q)$, (b) $\mathrm{Ca}(\mathrm{OH})_{2}(a q)$. 


\section{Summary and conclusions}

A two-scale approach to reaction-diffusion problems in porous materials was applied to a simplified situation related to the carbonation of concrete. In contrast to common purely macroscopic models, the two-scale model is capable of resolving processes on the microscopic (pore) scale. As a first approach, two different, highly simplified RUC geometries were considered.

With respect to the carbonation problem, the two-scale approach appears promising as there are microscopic effects which are not captured by purely macroscopic models. For a spherical pore configuration, results of the corresponding purely macroscopic model can be recovered. The predicted carbonation depths are in the range of experimental data. Strong delay effects due to processes on the micro scale are obtained if a pore geometry based on narrow pore channels is assumed and if the diffusivity of the species in the pore water is sufficiently small. Although this special setting does not seem to be realistic for the problem of concrete carbonation, the results confirm the capability of the two-scale model of resolving processes on the pore scale. The structure of the RUC allows to account for the microstructure of the material which can strongly affect the macroscopic output of the model.

It can be expected that the two-scale model can better account for microscopic effects like different types of cements, changes of the microstructure due to carbonation and the distribution of pore water inside the pores, all of which have been neglected in the present study.

The essential constraint of the two-scale approach is the increased computational effort in contrast to the pure macromodel. However, efficient algorithms exist for two-scale approaches in other research areas [9-11] and it can be expected that they can be adapted to this setting as well. Another challenge is the increased number of input parameters. Most of them require information on the pore geometry. Although the microstructure of concrete is well-studied, a reliable representation by an appropriate RUC is still a difficult problem due to its complexity.

\section{Acknowledgements}

The second author wishes to acknowledge the financial support of the German National Academic Foundation. 


\section{References}

[1] J. Kropp, Relations between transport characteristics and durability, in: J. Kropp, H. K. Hilsdorf (Eds.), Performance criteria for concrete durability, RILEM Report 12, E \& FN SPON, 1995, pp. 97-137.

[2] T. A. Bier, Karbonatisierung und Realkalisierung von Zementstein und Beton, Ph.D. thesis, University of Karlsruhe (1988).

[3] T. Chaussadent, États de lieux et réflexions sur la carbonatation du beton armé, Tech. Rep. LCPC OA29, Laboratoire Central de Ponts et Chaussées, Paris (1999).

[4] A. V. Saetta, B. A. Schrefler, R. V. Vitaliani, 2-D model for carbonation and moisture/heat flow in porous materials, Cem. Concr. Res. 25 (1995) 1703-1712.

[5] V. G. Papadakis, C. G. Vayenas, M. N. Fardis, A reaction engineering approach to the problem of concrete carbonation, AIChE J. 35 (10) (1989) 1639-1650.

[6] A. Steffens, D. Dinkler, H. Ahrens, Modeling carbonation for corrosion risk prediction of concrete structures, Cem. Concr. Res. 32 (2002) 935-941.

[7] T. Arbogast, J. Douglas Jr., U. Hornung, Derivation of the double porosity model of single phase flow via homogenization theory, SIAM J. Math. Anal. 21 (4) (1990) 823-836.

[8] R. E. Showalter, N. J. Walkington, Micro-structure models with diffusion in fissured media, J. Math. Anal. Appl. 155 (1991) 1-20.

[9] U. Hornung (Ed.), Homogenization and porous media, Springer, 1997.

[10] V. Kouznetsova, M. G. D. Geers, W. A. M. Brekelmans, Multi-scale constitutive modelling of heterogeneous materials with a gradient-enhanced computational homogenization scheme, Int. J. Numer. Meth. Eng. 54 (2002) 1235-1260.

[11] T. I. Zohdi, P. Wriggers, Introduction to computational micromechanics, Springer, 2005.

[12] R. Sander, Compilation of Henry's law constants for inorganic and organic species of potential importance in environmental chemistry, Tech. rep., Air Chemistry Department, Max-Planck Institute of Chemistry, Germany (1999).

[13] S. A. Meier, M. A. Peter, A. Muntean, M. Böhm, Modelling and simulation of concrete carbonation with internal layers, Berichte aus der Technomathematik 05-02, ZeTeM, University of Bremen (2005).

[14] U. Hornung, W. Jäger, Diffusion, convection, adsorption, and reaction of chemicals in porous media, J. Diff. Eq. 92 (1991) 199-225.

[15] H. I. Ene, D. Poliševski, Model of diffusion in partially fissured media, Z. angew. Math. Phys. 53 (2002) 1052-1059. 
[16] D. R. Lide (Ed.), CRC Handbook of Chemistry and Physics, 82nd Edition, CRC Press LLC, 2001.

[17] H.-J. Wierig, Longtime studies on the carbonation of concrete under normal outdoor exposure, in: Proceedings of the RILEM, Hannover University, 1984, pp. 239-249. 